\title{
Simulation of the adhesive-hard-sphere model
}

\author{
by W. G. T. KRANENDONK and D. FRENKEL \\ FOM Institute for Atomic and Molecular Physics, \\ P.O. Box 41883, 1009 DB Amsterdam, The Netherlands
}

(Received 28 August 1987; accepted 26 January 1988)

\begin{abstract}
Monte Carlo simulations of the three-dimensional sticky-hard-sphere system are presented. A new modified Monte Carlo algorithm has been developed, which makes it possible to explore the phase diagram for a large region of both the packing fraction and the stickiness parameter $\tau$. The phase diagram is calculated, as well as pair distribution functions and structure factors. Cluster percolation has been studied and its relation to the phase diagram. The simulation results are compared with predictions, obtained from the Percus-Yevick approximation, which can be solved analytically for this model. The potential relevance of the present simulation results for experiments on clustering in neutral colloids is discussed.
\end{abstract}

\section{Introduction}

The hard-sphere fluid is a useful model for dense, monatomic liquids. For such simple liquids, the experimental correlation functions and structure-factors agree quite well with both analytical results and computer simulations for hard spheres [1]. This is a reflection of the fact that the structure of simple fluids is dominated by the hard repulsion part of the intermolecular potential. Another interesting feature of the hard-sphere model is that it exhibits a fluid-solid transition [2]. The freezing of hard spheres, which is exclusively due to excluded volume effects, has been studied extensively both by computer simulation and, more recently, by density-functional theory [3].

Although the hard-sphere model has proved to be extremely useful, there remain many physically interesting properties of simple liquids that cannot be described in terms of a simple hard-core model. For example, attractive dispersion forces are usually invoked to explain the existence of a liquid-gas transition. Another phenomenon that requires strong attractive intermolecular interactions is the formation of clusters. In the present paper, we are interested in the phase behaviour of a model system in which the hard-core repulsion competes with short-range attractive interactions.

Of course, there are many possible model systems that combine a hard repulsive core with short-range attraction. For the sake of simplicity, we focus on the adhesive-sphere model ('sticky-hard-sphere model') first introduced by Baxter [4]. This model can be thought of as a special case of the square-well fluid in the limit where the well-depth goes to infinity while the well-width goes to zero, in such a way that the integral over the Boltzmann-factor in the region of the well yields a finite contribution to the second virial coefficient (see $\S 2$ ).

This particular model has a number of attractive features: first of all, as Baxter showed, the Percus-Yevick (PY) equation for this model can be solved in closed form. In the PY-approximation the adhesive-sphere model exhibits a 'gas-liquid' 
transition (see also [5, 6 and 7]). In addition, the adhesive-sphere system undergoes a solid-liquid transition $[8,19]$.

Following Baxter, other authors have explored the phase diagram of the sticky spheres, using different relations to calculate the pressure. In addition, generalizations of the sticky-sphere model have been investigated in studies of mixtures [7], competitive adhesion [9] and percolation [10].

Although at first sight the adhesive-sphere model appears rather pathological, it is a useful model system for real physical systems, such as colloidal suspensions of spherical particles with short range attractions. Hence we may hope that a study of the adhesive-sphere model will help us to understand the microscopic factors, that affect the tendency of a colloidal suspension to form a gel, a colloidal crystal or a dense fluid.

In contrast with the amount of theoretical work, little work has been done on computer simulation. In fact, the only numerical studies that we are aware of are due to Seaton and Glandt $[11,12,13,18]$. These authors employed a modified Monte Carlo method to calculate thermodynamical properties of the two-dimensional system of sticky hard discs and three-dimensional system of sticky hard spheres. Besides they studied the percolation behaviour of this system in three dimensions. Below we present the results of a Monte Carlo study of the 3D sticky-sphere model.

From the MC simulations we obtain structural properties such as the pair-distribution function $g(r)$ and the structure factor $S(k)$, which are then compared with the predictions resulting from the Percus-Yevick approximation.

This paper is organized as follows: in $\$ 2$ the adhesive-sphere model is briefly described. Our Monte Carlo algorithm is described in $\S 3$ and the technical details of the simulations are presented in $\$ 4$. The results for the correlation functions and percolation behaviour are discussed in $\S 5$.

\section{The adhesive-hard-sphere model}

The adhesive-hard-sphere potential can be thought of as a limiting case of the square-well potential, where the attractive well becomes infinitesimally narrow and infinitely deep. Whether such a model is meaningful depends crucially on the way in which this limit is taken. Baxter showed that the following choice of potential leads to a model with well-defined statistical mechanical properties.

$$
\begin{array}{rlrl}
\beta U & =\infty & \text { for } & 0<r<\sigma, \\
=\ln \left(\frac{12 \tau(d-\sigma)}{\sigma}\right), & & \sigma<r<d, \\
=0, & r>d,
\end{array}
$$

in the limit $d \rightarrow \sigma$, where $\sigma$ is the diameter of the particles. $\beta$ equals $1 / k T$, where $k$ is the Boltzmann-constant, $\tau$ is a measure of stickiness. Barboy has shown [6] that this parameter can be considered as a measure of the temperature.

Writing the potential as a sum of a hard sphere potential and an attractive well potential

$$
\beta U=\beta U_{\mathrm{hs}}+\beta U_{\mathrm{well}}
$$


with

$$
\begin{aligned}
\beta U_{\mathrm{hs}} & =\infty, & \text { for } & & r<\sigma \\
& =0, & & & r>\sigma
\end{aligned}
$$

and

$$
\beta U_{\text {well }}=\ln \left(\frac{12 \tau(d-\sigma)}{\sigma}\right), \quad \text { for } \quad \sigma<r<d
$$

we can express the canonical ensemble density of a system of $N$ identical particles as

$$
\begin{aligned}
\rho d r^{N} & =\lim _{d \rightarrow \sigma} \frac{\exp \left(-\beta\left\{U_{\mathrm{hs}}+U_{\text {well }}\right\} d \mathbf{r}^{N}\right.}{\exp \left(-\beta\left\{U_{\mathrm{hs}}+U_{\text {well }}\right\}\right) d \mathbf{r}^{N}} \\
& =\frac{(\sigma / 12 \tau)^{p} J_{p} \exp -\beta U_{\mathrm{hs}} d q_{p}}{Z},
\end{aligned}
$$

$r^{N}$ denotes all the translational degrees of freedom before taking the limit $d \rightarrow \sigma$, whereas $q_{p}$ represents all the degrees of freedom after taking this limit. So the set $q_{p}$ denotes all the translational degrees of freedom of both clusters and free particles as well as the internal and rotational degrees of freedom of the clusters. $J_{p}$ is the jacobian for the coordinate transformation from the cartesian coordinates $r^{N}$ to the generalized $q^{N}$.

$p$ is the number of particle-pairs with an interparticle distance between $\sigma$ and $d$ in the limit of $d \rightarrow \sigma$, so $p$ is in fact the number of 'bonds' in a configuration. The partition function $Z$ is given by

$$
Z=\sum_{p=0}^{p_{\text {max }}^{(N)}}\left(\frac{\sigma}{12 \tau}\right)^{p} \int \exp \left(-\beta U_{\mathrm{hs}}\right) J_{p} d q_{p},
$$

with $p_{\max }^{(N)}$ the maximum number of bonds in a system of $N$ particles.

From equation (2.5) for the density function it is clearly seen that the total phase-space consists of $p_{\max }^{(N)}+1$ disjunct subspaces, each subspace characterized by the number of bonds $p$.

We can compare this adhesive-sphere potential with other model potentials by use of the second virial coefficient [6]. The second virial coefficient is given by [14]

$$
B_{2}^{*}=4-\tau^{-1} \quad \text { with } \quad B_{2}^{*}=B_{2} / \sigma^{3},
$$

while the third virial coefficient is

$$
B_{3}^{*}=10-5 \tau^{-1}+\tau^{-2}-\tau^{-3} / 18,
$$

with

$$
B_{3}^{*}=B_{3} / \sigma^{6}
$$

\section{Monte Carlo sampling}

In order to compute canonical averages for the adhesive-sphere model by Monte Carlo simulation, we need an algorithm to sample all accessible configurations for a fixed number of bonds and, in addition, to sample over all possible number of bonds. 


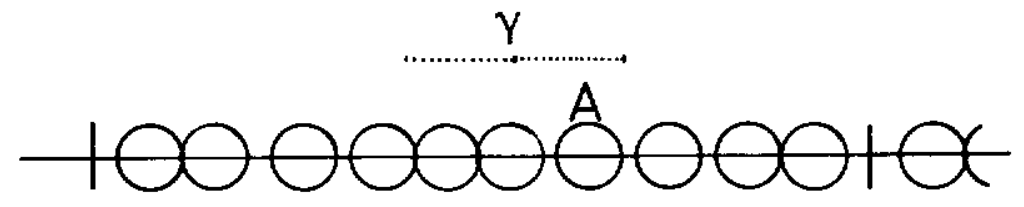

Figure 1. Schematic drawing of a (periodic) one-dimensional system of 'sticky spheres'. $A$ is the test particle and $\gamma$ is the random chosen centre. The dashed line under the spheres denotes the test line segment centred at $A$, the dotted line above the spheres denotes the test line segment centred at the random chosen centre $\gamma$.

In our Monte Carlo program a trial step consists either of an attempted displacement of a particle (or cluster of particles) at a fixed number of bonds or of an attempt to change the number of bonds. As the former type of trial move is straightforward we limit our discussion to the latter.

For the sake of simplicity we consider only a one-dimensional system in this section. The generalization for three dimensions is given in the Appendix. Figure 1 gives a view of such a one-dimensional system, consisting of free particles and clusters. We assume periodical boundaries in this example and in the three dimensional case later on.

We assume that the intermolecular potential has the form

$$
\begin{array}{rlrl}
\beta U & =\infty, & \text { for } & 0<r<\sigma, \\
=\ln \left(\frac{12 \tau(d-\sigma)}{\sigma}\right), & & \sigma<r<d, \\
=0, & r>d,
\end{array}
$$

with $d \rightarrow \sigma$.

The algorithm to change the number of bonds, works as follows. Consider a particle which can be chosen randomly. The centre of the particle defines the middle of a line segment of length $\Delta$, on which we randomly choose a second point. This second point is the centre of a second line segment with the same length as the first one. If we had chosen $\boldsymbol{A}$ for example (figure 1) the dashed line denotes the first mentioned line segment and the dotted line the second mentioned one, where $\gamma$ is the randomly chosen point.

On the latter line segment we randomly pick a new trial position for the particle under consideration. The reason for choosing such a seemingly complex method to generate trial moves is discussed below.

In the new configuration the particle may or may not form a bond with another particle on the line segment. Whether such a bond is possible depends on the local arrangement of the particles around the test particle. The probability for particle $A$ to form a bond in a given interval $\Delta$, for a fixed configuration of all other particles, is determined by the contribution of the configurational integral

$$
Q_{A}=\Delta+n\left(\frac{\sigma}{12 \tau}\right)
$$

where $\Delta$ is the contribution from the unbounded state, while everyone of the $n$ possible bonds in the interval $\Delta$ contributes a factor $(\sigma / 12 \tau)$. 
Using this notation, the probability to form a bond in this interval is

$$
P_{b}=\frac{n(\sigma / 12 \tau)}{\Delta+n(\sigma / 12 \tau)},
$$

while the probability to form no bonds is

$$
P_{\mu}=\frac{\Delta}{\Delta+n(\sigma / 12 \tau)} .
$$

Note that $(\sigma / 12 \tau)$ has the dimension of a (one-dimensional) volume. This allows us to interpret $(\sigma / 12 \tau)$ as the 'effective volume' available for one bond, and $\Delta+n(\sigma /$ $12 \tau)$ as the total effective volume in a segment of length $\Delta$.

The main difference with the $3 \mathrm{D}$ case is that in the latter case the probability to form a bond depends on the intersection of a sphere with the test sphere. In addition, in 3D one should also consider processes in which two or three bonds are formed simultaneously.

Returning to our one-dimensional example, we calculate the effective volume, $V_{\text {eff }}^{(i)}$, for each possible bond, in the way described above. We now randomly select a particular bond (i) with a probability $P(i)$ given by

$$
P(i)=\frac{V_{\text {eff }}^{(i)}}{\sum_{i} V_{\text {eff }}^{(i)}},
$$

where $i$ denotes the number of distinct bound or unbound configurations available to the particle under consideration within the test-line-segment. Note that the acceptance of a trial move depends only on the effective volumes in the new configuration. Hence this part of the program is rather different from the conventional (Metropolis) sampling scheme where the relative probabilities of the old and the new configurations must be compared. However, as will be discussed below, microscopic reversibility is maintained at all times.

After selecting a particular bound or unbound trial configuration we test for overlap and reject the trial move when a hard-core overlap is detected.

The correct definition of the line segment is of crucial importance to guarantee microscopic reversibility. Suppose we should use the conventional method: displacing in a line-segment centred at the original position of the test particle. If we should use the same method of choice for the line segment in the new position, the length (i.e. the effective volume of no bonds) of this second line segment would be the same as that around the original position. However the other effective 'volumina', due to the existing bonds, associated with both line segments would be different in most cases and therefore the probabilities of the forward and the reverse moves will be different. This violates the principle of microscopic reversibility. This problem is also discussed at length by Seaton and Glandt [11], however, the solution proposed by these authors differs from the one adopted here: as mentioned before, we first randomly select a point on the line segment. This randomly chosen point will be the centre of a (second) line segment, with the same length as the original one. On this last line segment the displacement takes place.

If we use the same method of defining a random-centre-line segment, both for the old and the new position, the method is reversible, because the probability to choose the same random-centre-line segment is the same in the old position and the new position, as are the associated effective 'volumina'. Furthermore, the method is quite efficient, as we only deal with line segments, and the calculation of the effective 
'volumina' remains easy. The extra work involved in the choice of a random centre is negligible.

In three dimensions the method is essentially the same. In a test-sphere of radius $\Delta$ centred at the position of the test particle a point is randomly selected. This point is the centre of a second sphere also with a radius $\Delta$, in which the displacement takes place. For a sphere, the calculation of the effective 'volumina' is relatively easy, as explained in the Appendix.

It seems that the present algorithm is more efficient than the algorithm proposed by Seaton and Glandt, especially at high densities [11]. The reason is the following: Seaton and Glandt allow trial moves to any point in the periodic box. The acceptance of such trial moves will be low at high densities. Because we use a restricted displacement by use of a random-centre-sphere, we can adapt the radius of this sphere to the density.

Secondly, the total number of computations (comparing the same number of particles under the same thermodynamic conditions) in our method will be much smaller than in the method of [11], since we only have to calculate the effective 'volumina' in a test-sphere, whereas in [11] these 'volumina' must be computed for the whole box. However, we have not attempted any direct comparison between our approach and the one employed by Seaton and Glandt.

To get a more efficient sampling of the configuration-space, we also performed trial displacements of all clusters. For such a displacement we regard a cluster as rigid. The magnitude of the attempted displacement depends on the number of particles in a cluster: the more the particles, the smaller the attempted displacement. We do not attempt to move a percolated cluster.

The structure factor $S(k)$ was calculated directly during the simulation by a fast Fourier transform of the density. So we compute directly

$$
\left.\begin{array}{rl}
\rho & =\sum_{j=1}^{N} \exp \left(-i \mathbf{k} \cdot \mathbf{r}_{j}\right), \\
S(k) & =\frac{1}{N}\langle\rho(k) \rho(-k)\rangle,
\end{array}\right\}
$$

with $N$ the numbers of particles. In the transform, we take a spatial resolution of 256 points in the direction of $k$. This number is sufficient to suppress aliasing effects. In order to improve our statistics we compute $\rho(k)$ for $k$ along 13 independent directions (3 along $P([100]), 6$ along $P([110])$ and 4 along $P([111])$, where $P$ stands for all independent permutations).

The $13 \times 4$ Fourier transforms were carried out every 10 sweeps, which resulted in a negligible increase in computer time. The pair distribution function $g(r)$ was calculated by keeping a histogram of the interparticle distances. Because we measured the average over finite bins there is some loss of accuracy at points where $g(r)$ has singularities, except for the singularity at $r=\sigma$, which was computed explicitly by counting the number of bonds.

\section{Monte Carlo simulations}

Constant pressure simulations were carried out on model systems consisting of 32 particles. Constant volume simulations were done on systems of 32,108 and 256 particles. The 'stickiness' parameter $\tau$ was varied between 0.05 and $\infty$ (hard spheres). 
typical duration of a Monte Carlo run was 1-2 $10^{4}$ trial moves per particle (excluding equilibration) for the 32 and 108 particle systems, and $210^{3}$ cycles for the 256 particle system. The 256 particle systems were equilibrated for $10^{3} \mathrm{cycles}$, but this number excludes the equilibration of the 32 particle system from which the 256 particle system was prepared by doubling the linear dimensions of the periodic box. On a Cyber 750-855, a typical run on a 256 particle system took 1-2 hours, but most runs on the smaller systems took appreciably less time.

The equation-of-state data were obtained from the constant pressure MC simulations. This method, although straightforward, has the disadvantage that it becomes inefficient at low temperatures and high densities. We therefore also experimented with methods to derive the pressure from the value of the pair-correlation function at contact, as obtained in constant density runs. One such method was used by Seaton and Glandt [11] in their simulation of sticky discs in two dimensions. This method is based on an extension of the relation between the pressure of a square-well fluid and the value of the radial distribution function inside the well, as used by Rotenberg [15]. It relates the pressure of the sticky-disc (sticky-sphere) fluid to the average number of bonds per particle

$$
\frac{\beta P}{\rho}=1+4\langle n\rangle\left(\tau-\frac{1}{4}\right),
$$

where $\langle n\rangle$ is the mean number of bonds per particle.

Equation (4.1) is based on the assumption that $y(r) \equiv \exp (\beta u(r)) g(r)$ is continuous through the attractive well. We found that equation (4.1) leads to incorrect values of the pressure, even at relatively low densities, especially when there exists a percolated cluster. Other methods to compute the pressure in constant density runs either suffered from the same drawback, or were computationally inefficient. For this reason, we were forced to rely exclusively on the constant-pressure simulations to measure the sticky-sphere equation-of-state.

The main reason why the constant pressure method becomes inefficient (although, in principle, still correct) at high densities and low temperatures is that under these conditions percolation may take place. Percolation in a periodic system means that there is at least one cluster in the simulation box which is connected to its periodic image. Once such a cluster has formed, it is impossible to change the volume of the box without either creating a hard-core overlap or breaking a bond. But volume-changes moves are essential for the constant-pressure Monte Carlo simulation. Hence, as the probability of percolation increases, the efficiency of constant-P MC goes down. This limitation is most serious in the larger systems, where the percolation transition becomes quite sharp. It is least serious for the simulations of the 32-particle system, because for such a small system-size the percolation transition is not very sharp. As a consequence, it is still possible to find unpercolated configurations at temperatures and densities that are well beyond the percolation transition of the infinite system.

Apart from the problem with percolation at low $\tau$ and high $\phi$, there is the additional problem that at very low $\tau$ (typically, $\tau \leqslant 0 \cdot 1$ ), uniform sampling of configuration space becomes increasingly difficult. Under these conditions, the measured properties of the system become strongly dependent on its previous history. For instance, different quenches from a high to a low value of $t$ tend to result in different inhomogeneous 'glassy' structures that hardly relax in time. Although such behaviour is usually observed in low-temperature percolated structures, it is not due 


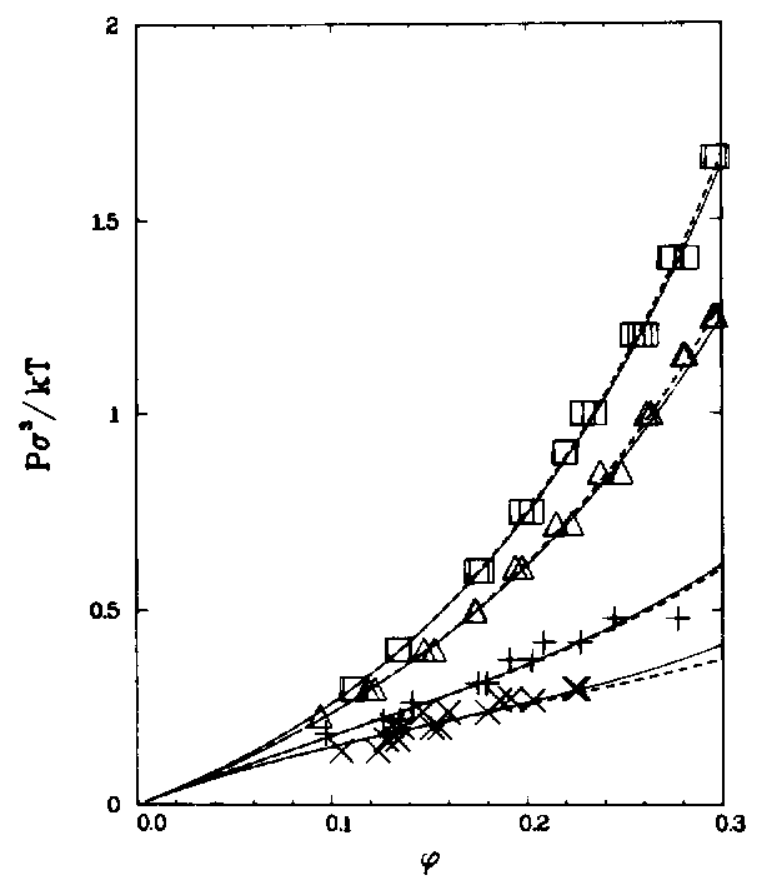

Figure 2. Phase diagram of the three-dimensional sticky-hard-sphere system. Horizontal axis: the packing fraction $\phi$, vertical axis: pressure $P \sigma^{3} / k T . \times, \tau=0.15 ;+, \tau=0.2$; $\triangle, \tau=0.5 ; \square, \tau=1$. Number of particles: 32 . Percus-Yevick predictions are indicated by full lines (compressibility equation) and dashed lines (energy equation).

to percolation as such. In fact, for $\tau=0.2$, configuration space can be sampled quite efficiently even for packing fractions as high as $\phi=0 \cdot 4$, although this is well inside the percolation regime.

\section{Results}

\subsection{Structural properties}

Figure 2 shows the constant-pressure Monte Carlo results for the equation of state of the sticky-hard-sphere fluid. The data have been collected in table 1 . In this table we also indicate the length of the simulation. In figure 2, the MC results are compared with the predictions of the Percus-Yevick approximation, using both the compressibility relation [4] (solid line) and the energy relation [5] (dashed line). For high $\tau$ both relations give almost the same result. Only near the critical isotherm, large differences between the results of both relations occur [5].

Note that, over the range of densities and temperatures that could be studied by constant- $P$ MC the Percus-Yevick approximation agrees quite well with the simulation results. At higher densities and lower temperatures we can no longer obtain reliable equation-of-state data, but we can measure the radial distribution function $g(r)$. The latter quantity can also be compared with the corresponding PercusYevick prediction. Such a comparison is shown in figures 3-6, where the particle separation $r$ is in units of the sphere diameter $\sigma$. The radial distribution function in the PY approximation was calculated by Fourier-transforming the structure factor 
Table 1. Pressure as a function of the packing fraction. $\tau$ denotes the stickiness or 'temperature', while $\phi$ denotes the packing fraction. $N_{\text {equi }}$ is the number of MC sweeps to relax the system to equilibrium and $N_{\text {samp }}$ is the number of MC sweeps used to calculate the thermodynamic function.

\begin{tabular}{ccllr}
\hline$\tau$ & \multicolumn{1}{c}{$\phi$} & \multicolumn{1}{c}{$P$} & \multicolumn{1}{c}{$N_{\text {equi }}$} & \multicolumn{1}{c}{$N_{\text {samp }}$} \\
\hline $0 \cdot 15$ & $0 \cdot 1051$ & $0 \cdot 14$ & 4000 & 15000 \\
$0 \cdot 15$ & $0 \cdot 1239$ & $0 \cdot 14$ & 4000 & 10954 \\
$0 \cdot 15$ & $0 \cdot 1280$ & $0 \cdot 17$ & 4000 & 11125 \\
$0 \cdot 15$ & $0 \cdot 1310$ & $0 \cdot 2073$ & 2000 & 11733 \\
$0 \cdot 15$ & $0 \cdot 1341$ & $0 \cdot 17$ & 4000 & 12706 \\
$0 \cdot 15$ & $0 \cdot 1363$ & $0 \cdot 2$ & 4000 & 11007 \\
$0 \cdot 15$ & $0 \cdot 1467$ & $0 \cdot 2407$ & 2000 & 15000 \\
$0 \cdot 15$ & $0 \cdot 1516$ & $0 \cdot 2$ & 4000 & 4379 \\
$0 \cdot 15$ & $0 \cdot 1555$ & $0 \cdot 2$ & 4000 & 11316 \\
$0 \cdot 15$ & $0 \cdot 1599$ & $0 \cdot 24$ & 4000 & 10775 \\
$0 \cdot 15$ & $0 \cdot 1800$ & $0 \cdot 24$ & 4000 & 9793 \\
$0 \cdot 15$ & $0 \cdot 1861$ & $0 \cdot 27$ & 4000 & 15000 \\
$0 \cdot 15$ & $0 \cdot 1917$ & $0 \cdot 2757$ & 2000 & 14434 \\
$0 \cdot 15$ & $0 \cdot 2035$ & $0 \cdot 27$ & 4000 & 13174 \\
$0 \cdot 15$ & $0 \cdot 2247$ & $0 \cdot 3$ & 4000 & 15000 \\
$0 \cdot 15$ & $0 \cdot 2263$ & $0 \cdot 3$ & 4000 & 15000 \\
$0 \cdot 2$ & $0 \cdot 0974$ & $0 \cdot 185$ & 2000 & 15000 \\
$0 \cdot 2$ & $0 \cdot 1268$ & $0 \cdot 22$ & 4000 & 13607 \\
$0 \cdot 2$ & $0 \cdot 1353$ & $0 \cdot 22$ & 4000 & 12419 \\
$0 \cdot 2$ & $0 \cdot 1416$ & $0 \cdot 265$ & 2000 & 14274 \\
$0 \cdot 2$ & $0 \cdot 1751$ & $0 \cdot 315$ & 4000 & 15000 \\
$0 \cdot 2$ & $0 \cdot 1797$ & $0 \cdot 315$ & 2000 & 15000 \\
$0 \cdot 2$ & $0 \cdot 1915$ & $0 \cdot 375$ & 2000 & 9975 \\
$0 \cdot 2$ & $0 \cdot 2029$ & $0 \cdot 375$ & 2000 & 14076 \\
$0 \cdot 2$ & $0 \cdot 2091$ & $0 \cdot 42$ & 4000 & 15000 \\
$0 \cdot 2$ & $0 \cdot 2277$ & $0 \cdot 42$ & 4000 & 15000 \\
$0 \cdot 2$ & $0 \cdot 2451$ & $0 \cdot 48$ & 2000 & 9233 \\
$0 \cdot 2$ & $0 \cdot 2774$ & $0 \cdot 48$ & 2000 & 11588 \\
$0 \cdot 5$ & $0 \cdot 0943$ & $0 \cdot 23$ & 2000 & 15000 \\
$0 \cdot 5$ & $0 \cdot 1187$ & $0 \cdot 3$ & 4000 & 3929 \\
$0 \cdot 5$ & $0 \cdot 1224$ & $0 \cdot 3$ & 4000 & 13177 \\
$0 \cdot 5$ & $0 \cdot 1475$ & $0 \cdot 4$ & 4000 & 6167 \\
$0 \cdot 5$ & $0 \cdot 1528$ & $0 \cdot 4$ & 4000 & 15000 \\
$0 \cdot 5$ & $0 \cdot 1736$ & $0 \cdot 5$ & 4000 & 15000 \\
$0 \cdot 5$ & $0 \cdot 1740$ & $0 \cdot 5$ & 4000 & 14270 \\
$0 \cdot 5$ & $0 \cdot 1943$ & $0 \cdot 61$ & 2000 & 15000 \\
$0 \cdot 5$ & $0 \cdot 1978$ & $0 \cdot 61$ & 2000 & 15000 \\
$0 \cdot 5$ & $0 \cdot 2154$ & $0 \cdot 72$ & 4000 & 15000 \\
$0 \cdot 5$ & $0 \cdot 2233$ & $0 \cdot 72$ & 4000 & 15000 \\
$0 \cdot 5$ & $0 \cdot 2378$ & $0 \cdot 85$ & 4000 & 15000 \\
$0 \cdot 5$ & $0 \cdot 2487$ & $0 \cdot 85$ & 4000 & 15000 \\
$0 \cdot 5$ & $0 \cdot 2616$ & $1 \cdot 0$ & 4000 & 15000 \\
$0 \cdot 5$ & $0 \cdot 2638$ & $1 \cdot 0$ & 4000 & 15000 \\
$0 \cdot 5$ & $0 \cdot 2803$ & $1 \cdot 15$ & 4000 & 10901 \\
$0 \cdot 5$ & $0 \cdot 2816$ & $1 \cdot 15$ & 4000 & 15000 \\
$0 \cdot 5$ & $0 \cdot 2954$ & $1 \cdot 25$ & 2000 & 15000 \\
$0 \cdot 5$ & $0 \cdot 2969$ & $1 \cdot 25$ & 2000 & 15000 \\
$1 \cdot 0$ & $0 \cdot 1106$ & $0 \cdot 3$ & 2000 & 15000 \\
$1 \cdot 0$ & $0 \cdot 1124$ & $0 \cdot 3$ & 4000 & 15000 \\
$1 \cdot 0$ & $0 \cdot 1344$ & $0 \cdot 4$ & 4000 & 15000
\end{tabular}


Table 1 (continued).

\begin{tabular}{cclcc}
\hline$\tau$ & $\phi$ & \multicolumn{1}{c}{$P$} & $N_{\text {equi }}$ & $N_{\text {samp }}$ \\
\hline 1.0 & 0.1348 & 0.4 & 4000 & 11053 \\
1.0 & 0.1746 & 0.6 & 4000 & 15000 \\
1.0 & 0.1771 & 0.6 & 4000 & 15000 \\
1.0 & 0.1976 & 0.75 & 4000 & 15000 \\
1.0 & 0.2029 & 0.75 & 2000 & 15000 \\
1.0 & 0.2200 & 0.90 & 4000 & 15000 \\
1.0 & 0.2204 & 0.90 & 4000 & 15000 \\
1.0 & 0.2293 & 1.0 & 4000 & 15000 \\
1.0 & 0.2351 & 1.0 & 4000 & 15000 \\
1.0 & 0.2547 & 1.2 & 4000 & 15000 \\
1.0 & 0.2585 & 1.2 & 2000 & 15000 \\
1.0 & 0.2615 & 1.2 & 4000 & 15000 \\
1.0 & 0.2737 & 1.4 & 4000 & 15000 \\
1.0 & 0.2748 & 1.4 & 4000 & 15000 \\
1.0 & 0.2823 & 1.4 & 4000 & 15000 \\
1.0 & 0.2959 & 1.66 & 2000 & 15000 \\
1.0 & 0.2979 & 1.66 & 4000 & 15000 \\
\hline
\end{tabular}

in the PY approximation. The structure factor can be calculated by Fourier-transforming the Percus-Yevick direct correlation function $c(r)$ [4]. Below $\tau=(\sqrt{ } 2-1) / \sqrt{ } 18$ there is a range of densities (inside the liquid-vapour coexistence region) where no real solutions for $c(r)$ exist.

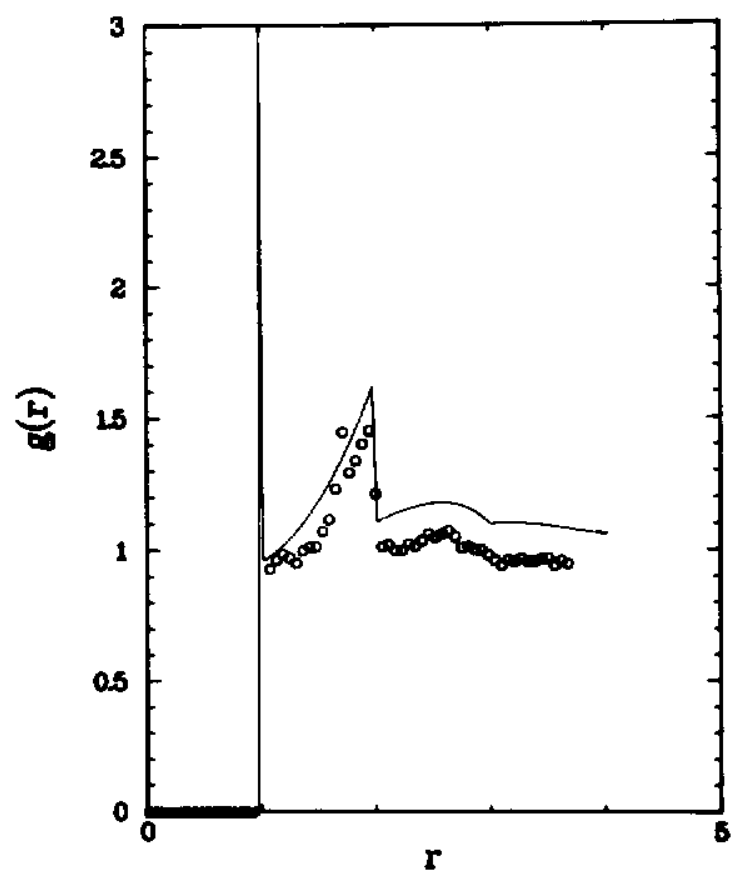

Figure 3. Pair distribution function $g(r)$ versus $r$ in units of the particle diameter $\sigma$. 'Temperature' $\tau=0 \cdot 1$, packing fraction $\phi=0 \cdot 14$. System size: $N_{\text {part }}=108$. Number of MC-sweeps to relax the system to equilibrium: $N_{\text {equi }}=5000$, number of MC-sweeps to calculate the correlation function: $N_{\mathrm{samp}}=9700$. Full line is the PY prediction: dots represent the $\mathrm{MC}$ results. 


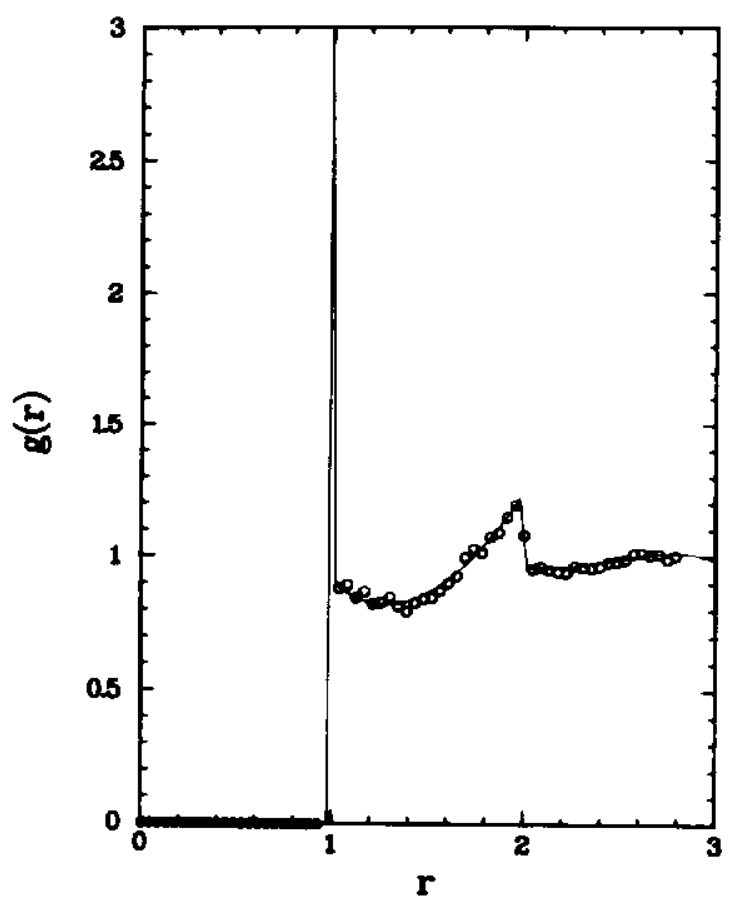

Figure 4. Meaning of symbols as in figure 3. $\tau=0 \cdot 2 ; \phi=0 \cdot 32 . N_{\text {part }}=108 . N_{\text {equi }}=5000$;

$$
N_{\text {samp }}=8000 \text {. }
$$

The radial distribution functions shown in figures 3-6 were computed in constant-density runs. Looking at figures $3-6$ it is clear that the Percus-Yevick expression for the radial distribution function is in good overall agreement with the Monte Carlo data for $0.15<\tau<1$, and $0<\phi<0.5$. It is noteworthy that even for $\tau=1$, and $\phi=0.50$ (figure 6), the PY approximation for $g(r)$ is indistinguishable from the simulation results. This contrasts with the behaviour for $\tau=\infty$ (hard spheres), for which case there are clear deviations between $g(r)$ obtained by computer simulation and the corresponding PY predictions [16].

Serious discrepancies between the PY approximation and the simulation results show up for $\tau \leqslant 0 \cdot 1$ (figure 3 ), although there is clearly still qualitative agreement.

A peculiar feature of the radial distribution function of sticky spheres is the occurrence of discontinuities in $g(r)$ or its derivatives at distances, which are a multiple of the hard-core diameter. This behaviour is a result of the delta-function peak in $g(r)$ at $r=\sigma[17]$. For $\tau$ larger than $0 \cdot 15$, these discontinuities are reproduced to within the statistical accuracy of our calculations by the corresponding PY approximation for all packing fractions studied in our simulations.

However, the Percus-Yevick approximation fails to account for positional correlations associated with the presence of larger rigid clusters such as the trigonal bipyramid. Such structures are formed by attaching additional particles with three bonds to a tetrahedral cluster. These rigid clusters give rise to an infinite series of delta-functions in $g(r)$, often at irrational values of $r$. For example, the distance between the two particles at the 'top' and 'bottom' of the trigonal pyramid is $\sqrt{ }(8 / 3)$. These contributions will show up in the pair distribution functions, especially at low $\tau$ (figure 3). In this figure, each delta function is smeared out over an 


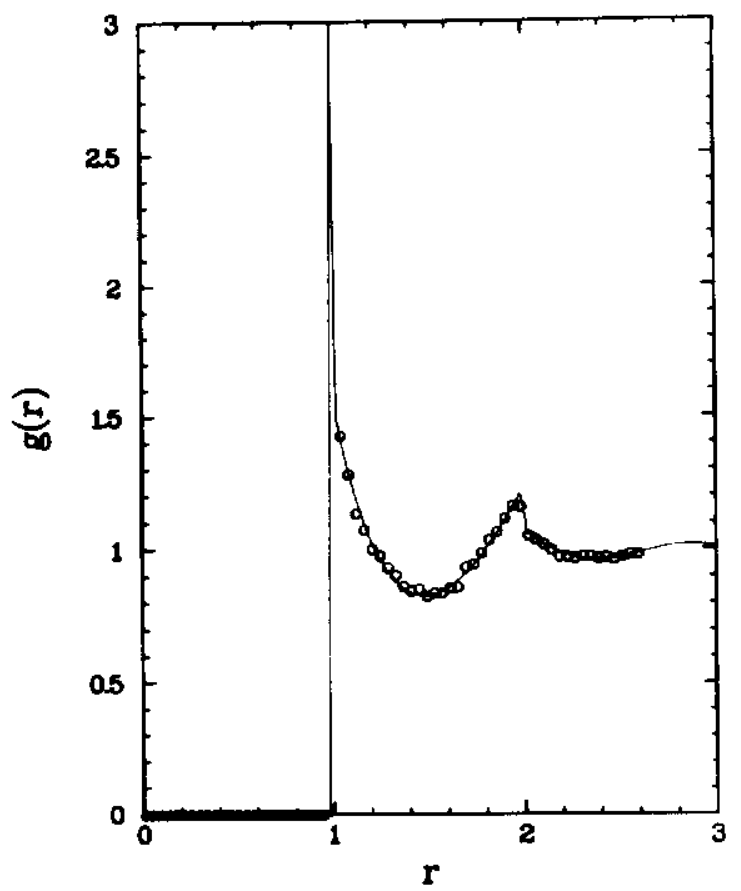

Figure 5. Meaning of symbols as in figure 3. $\tau=0.5 ; \phi=0 \cdot 40 . N_{\text {part }}=108 . N_{\text {equi }}=5000$; $N_{\text {samp }}=10000$.

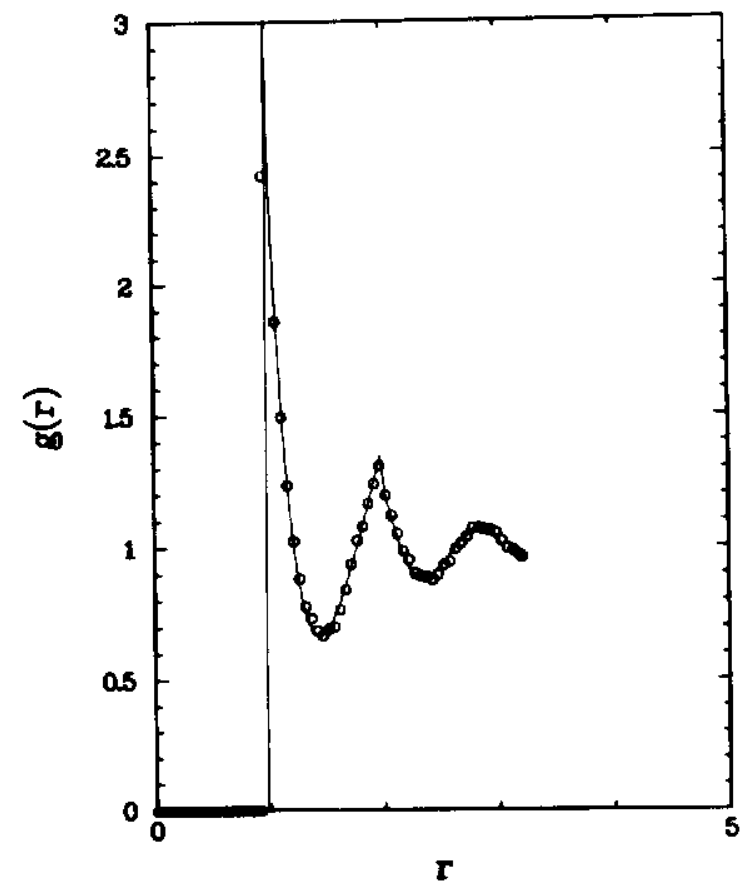

Figure 6. Meaning of symbols as in figure 3. $\tau=1 ; \phi=0.50 . N_{\text {part }}=256 . N_{\text {equi }}=500$; $N_{\text {samp }}=2000$. 


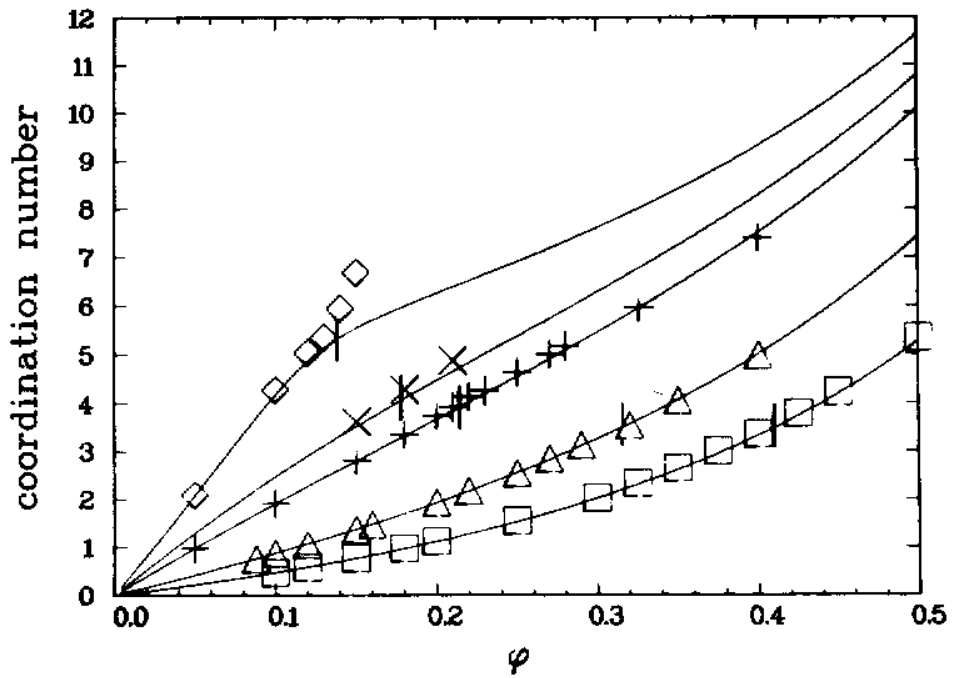

Figure 7. Mean coordination number as a function of the packing fraction $\phi, \diamond, \tau=0 \cdot 1$; $x, \tau=0 \cdot 15 ;+, \tau=0 \cdot 2 ; \Delta, \tau=0 \cdot 5 ; \square, \tau=1$. System size: 256 particles. The vertical bars denote the percolation thresholds for different values of $\tau$.

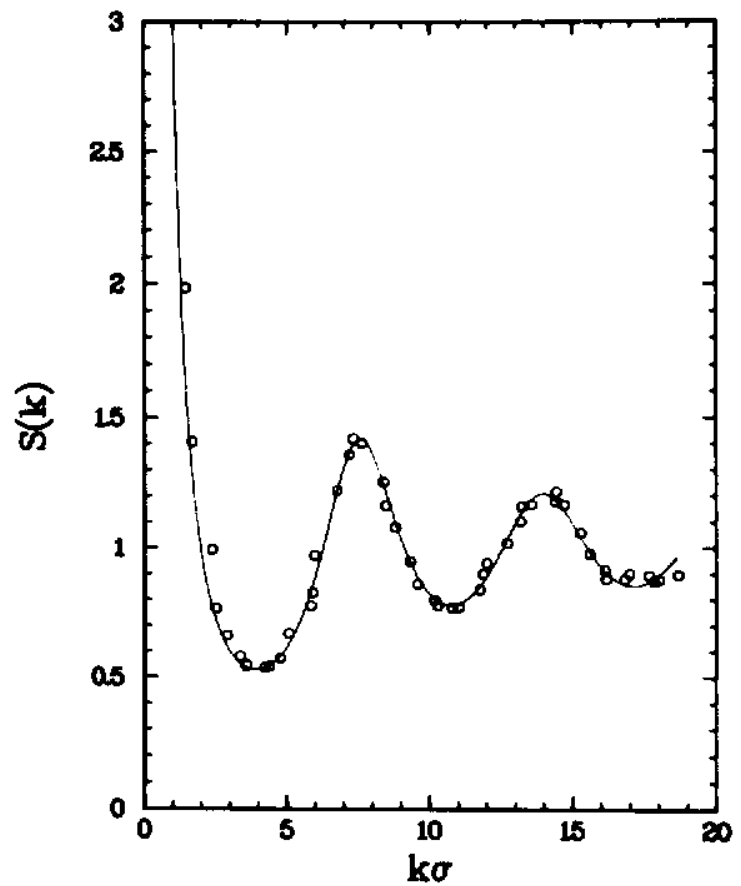

Figure 8. Structure factor $S(k)$ versus $k \sigma$ for the sticky-hard-sphere fluid. 'Temperature' $\tau=0 \cdot 1$; packing fraction $\phi=0 \cdot 14$. System size: $N_{\text {part }}=108$. Number of MC-sweeps to relax the system to equilibrium: $N_{\text {equi }}=5000$, number of MC-sweeps to calculate the correlation function: $N_{\text {samp }}=9700$. Full line: PY results; dots represent the MC results. 


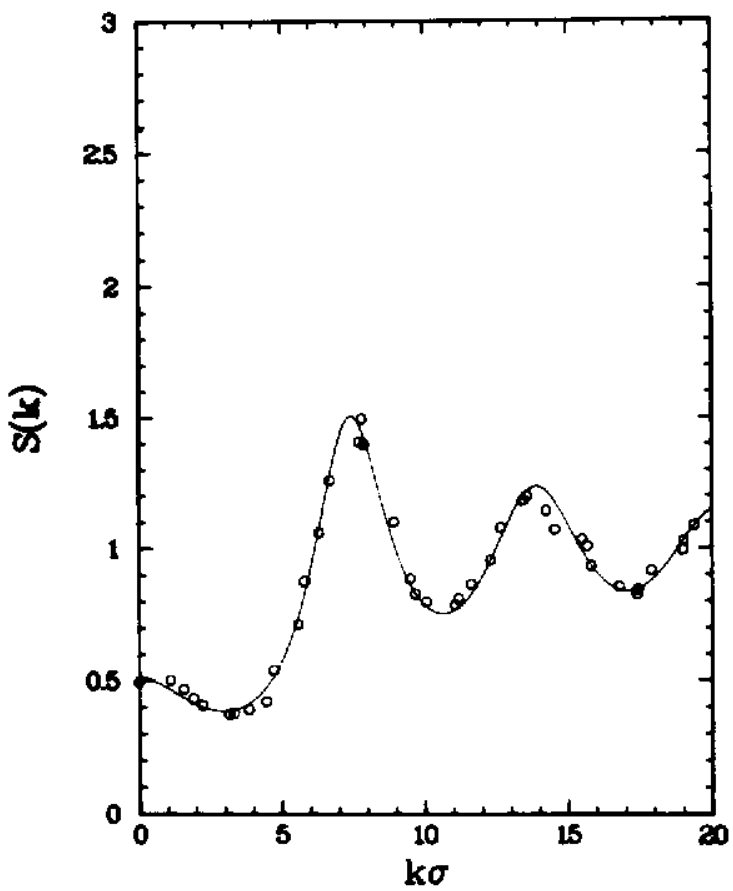

Figure 9. Meaning of symbols as in figure 8. $\tau=0 \cdot 2 ; \phi=0 \cdot 32 . N_{\text {part }}=108 . N_{\text {equi }}=5000$; $N_{\text {samp }}=8000$.

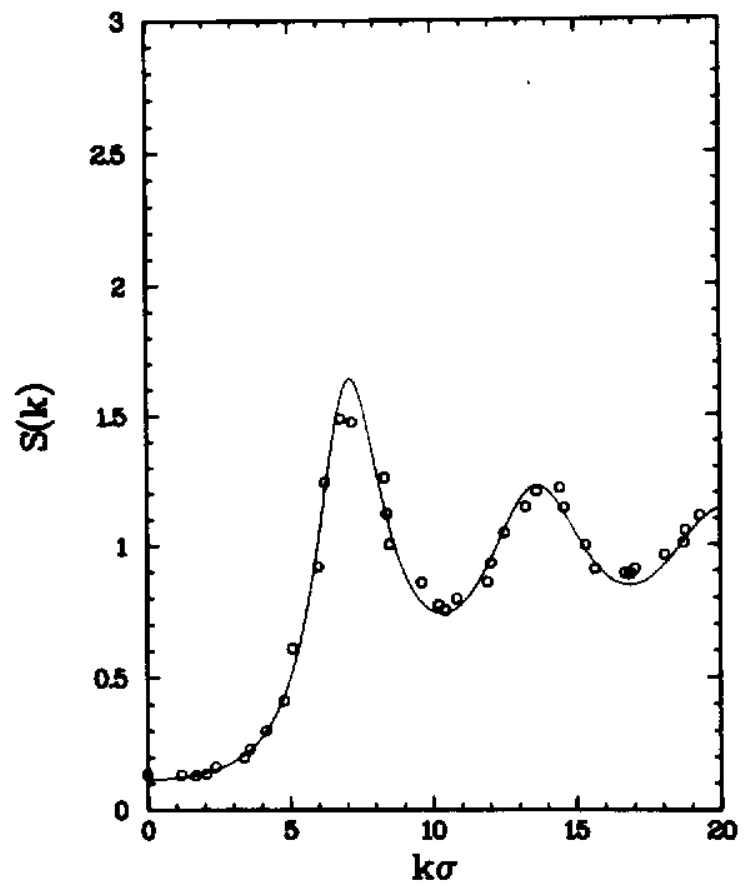

Figure 10. Meaning of symbols as in figure 8. $\tau=0 \cdot 5 ; \phi=0 \cdot 40 . N_{\text {part }}=108 . N_{\text {equi }}=5000$; $N_{\text {samp }}=10000$. 


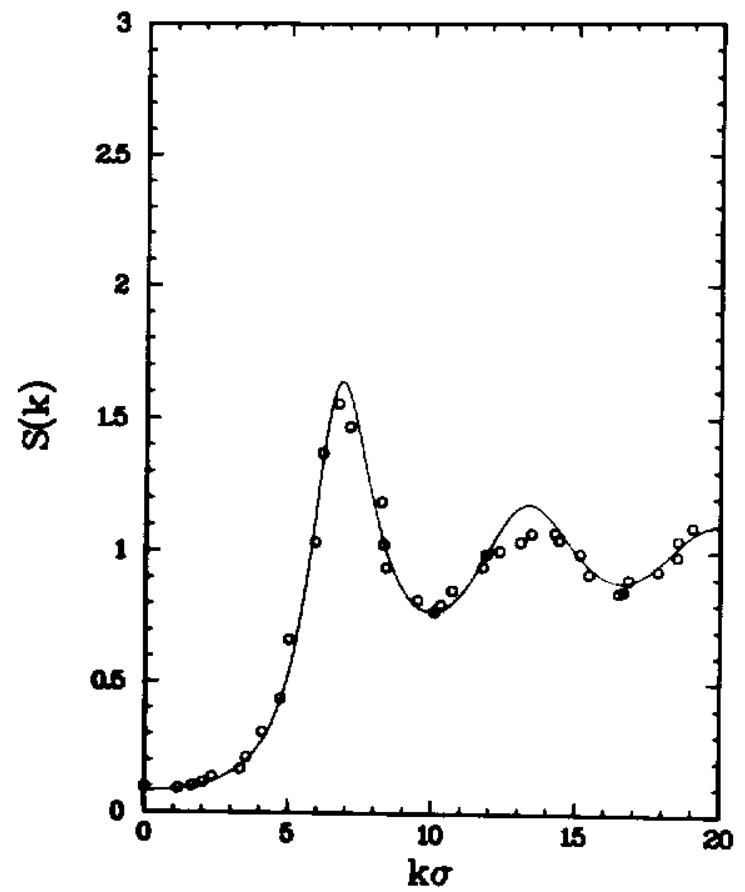

Figure 11. Meaning of symbols as in figure 8. $\tau=1.0 ; \phi=0.385, N_{\text {part }}=108, N_{\text {equi }}=5000$;

$$
N_{\text {samp }}=10000 \text {. }
$$

interval corresponding to the spatial resolution with which we measure $g(r)$. Therefore, only large contributions will show up as peaks in $g(r)$. An example is the peak at $r=1.634$ in figure 3 . These conclusions are in close agreement with those reached in a very recent publication by Seaton and Glandt [18]. These authors also found that the Percus-Yevick approximation works well at $\tau=0.2$, while at $\tau=0.1$ and $\phi=0.1$ the Percus-Yevick approximation of $g(r)$ is some 10 per cent too high, which agrees with our results shown in figure 3.

The value of $g(r)$ at $r=\sigma$ measures the mean number of bonds in a system of hard, adhesive spheres. This number can be compared with the PY prediction given by Baxter. The results of this comparison are shown in figure 7 . The mean coordination number, i.e. the mean number of particles sticking to a particular particle, is twice the mean number of bonds. The agreement between the PY approximation and the simulation results is again quite good. The PY approximation breaks down when the mean number of bonds per particle approaches the typical coordination number of a particle in a dense fluid. Note that the figure shows that the agreement between simulation and the Percus-Yevick prediction extends even beyond the percolation threshold. At low densities our results for the coordination number agree well with those obtained by Seaton and Glandt [13], if one takes into account that these authors use a definition of the coordination number that differs from the conventional one by a factor of two. At $\tau=0 \cdot 1$ and $\phi=0 \cdot 14$, we find a coordination number which is slightly larger than the PY estimate while the results of Seaton and Glandt are slightly lower. However, one should note that the latter state point is in or near the 'glassy' region in the phase diagram. Hence one should 
expect large statistical fluctuations due to incomplete sampling of the configuration space.

Examples of the calculated structure factors are displayed in figures 8-11.

At high values of $\tau$, the structure factor of sticky hard spheres resembles that of hard spheres. If the density is increased, while keeping $\tau$ constant, the amplitude of the oscillations in the structure factor grows, while the first maximum is shifted to slightly higher $k$ values.

Lowering the 'temperature' at constant density leads to a behaviour that is different from hard spheres. However, there is still good overall agreement between $S(k)$ obtained by simulation and the corresponding PY prediction.

Nevertheless, some differences between the analytical theory and the simulation results can be seen near the extrema of $S(k)$. In general the Percus-Yevick theory tends to underestimate the amplitude of the oscillations in $S(k)$. This failure cannot be explained as an underestimation of the pair distribution function at $r=\sigma$ in the PY theory. Figure 7 shows clearly that the PY approximation gives a very good estimate for the averaged coordination number at $\tau$ far from the critical point and even at high densities. Another possible explanation is that in case of high density (figures 10 and 11) the system is near a fluid-solid or fluid-metastable glass-transition. The results of an approximate density-functional calculation for sticky-hard spheres give some support for this assumption $[8,19]$.

A large increase of $S(k=0)$ takes place, when $\tau$ is lowered (figure 8$)$. $S(0)$ is a measure for the isothermal compressibility. The latter quantity becomes infinite at a critical point or, inside the two-phase region, on the spinodal line. The observed increase of $S(0)$ suggests the approach to a spinodal line. The 'temperature' at which we observe this increase in $S(0)$ agrees well with the (compressibility) PercusYevick estimate of the location of the spinodal line for low densities. Figure 8 illustrates the peak in $S(k)$ at $k=0$ that develops as the temperature is lowered. As can be seen from the figures, the peak in $S(k)$ is well described by the PY approximation.

Although the adhesive-sphere model is, of course, an oversimplification, it is expected to provide a qualitatitively correct description of a suspension of uncharged colloidal particles. It is interesting to note that recent small-angle neutron scattering experiments on suspension of uncharged colloidal particles do indeed show a divergence of the compressibility (or, equivalently, of $S(k)$ as $k \rightarrow 0$ ), as the temperature is lowered towards a spinodal. This is in qualitative agreement with the predictions of the adhesive-sphere model.

\subsection{Percolation behaviour}

Due to the short range attractive potential, the adhesive sphere model is especially suitable to study clustering and percolation. As the range of attraction is infinitesimal, the coordination number of a given particle can be defined unambiguously as the number of particles sticking to that particle.

As the adhesive-hard-sphere model can be solved analytically in the PercusYevick approximation, it is possible to compare the simulation data with analytical results for cluster properties such as the mean cluster size and percolation threshold.

Coniglio et al. [20] developed a Percus-Yevick-like analytical approach to calculate mean cluster sizes and percolation behaviour in fluid systems. This approach 


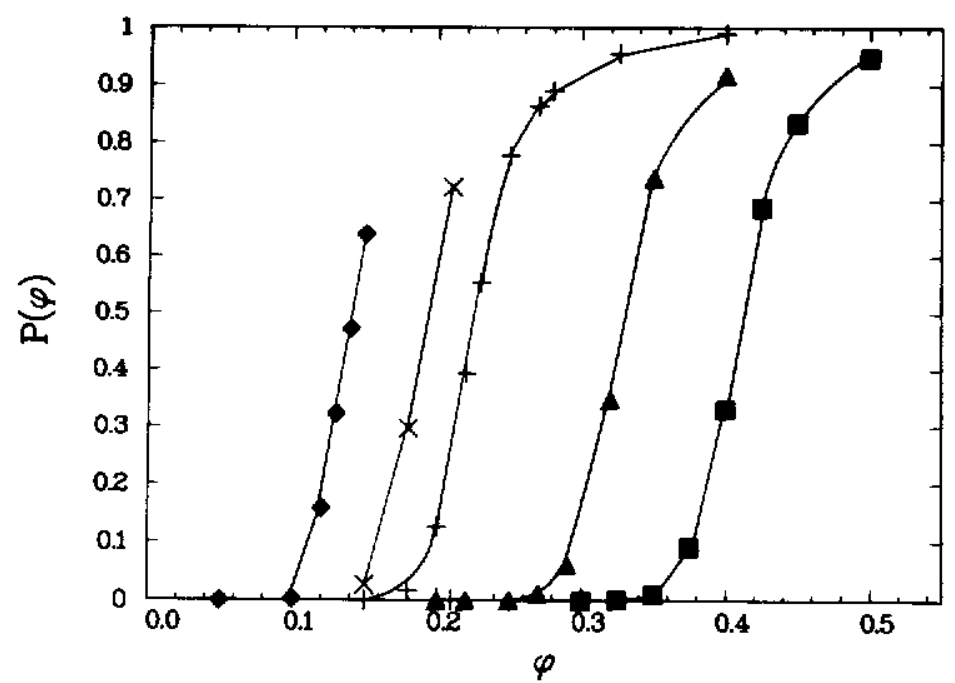

Figure 12. Percolation probability as a function of the packing fraction. Symbols: $\tau=0.1 ; \times, \tau=0.15 ;+, \tau=0.2 ; \boldsymbol{\Delta}, \tau=0.5 ; \boldsymbol{\square}, \tau=1$. The lines serve as a guide to the eye. System size is 256 particles.

was applied by Chiew and Glandt $[10]$ to estimate the percolation transition as a function of $\tau$ in the adhesive-sphere model.

In computer simulation the definition of percolation is determined by the period boundary conditions. One must find a cluster which links with its periodic images to form an infinite cluster.

In our algorithm for detecting percolating clusters, we examine all the particles subsequently. If a particle is already identified as belonging to a cluster, it is skipped. Each particle, that is not yet part of a previously determined cluster, is considered the seed of a new cluster. For such a seed, we next examine which particles, if any, are bonded to it. These particles are allocated to the same cluster as the seed. We then iterate the procedure for these new members of the cluster, until no new particles are found. We also keep track of the vectorial distances separating all particles in a given cluster. Of particular interest are all distinct loops joining one particle to itself. Two distinct situations may arise: either the vectorial distance between beginning and end of the loop is zero, in which case we have detected a ring in the cluster. Or the vectorial distance corresponds to a linear combination of lattice vectors of the periodic lattice.

With this algorithm we can detect all percolating clusters as well as the number of particles, that they contain. So we can calculate the percolation probability $P(\phi)$, i.e. the probability to find an 'infinite' cluster in the system. Plots of $P(\phi)$ for several different values of $\tau$ are shown in figure 12. The $P(\phi)$ as function of the packing fraction was calculated for $\tau=0.05,0.075,0 \cdot 1,0 \cdot 15,0.2,0.5,1$, for all the sample sizes, 32, 108 and 256 particles. Finite-size effects smear out the percolation transition, as can be seen clearly in figure 12 . It is therefore difficult to give a reliable estimate for the percolation threshold in the infinite system limit. In practice, however, it turns out that the point at which the percolation probability $P(\phi)=50$ per cent, is not very sensitive to system size (figure 13). This figure shows that there is only a slight shift in the percolation threshold with system size. We found this 


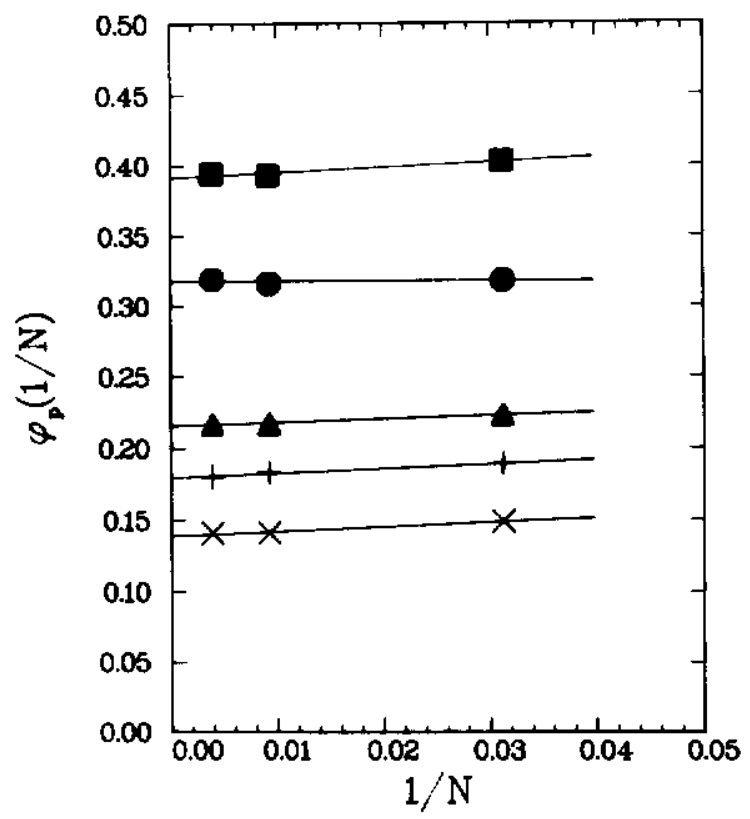

Figure 13. Extrapolation of the percolation thresholds of finite systems of sticky spheres to $N \rightarrow \infty . \phi_{p}(1 / N)$ denotes the packing fraction at the percolation threshold. Symbols: $\times, \tau=0.1 ;+, \tau=0.15 ; \boldsymbol{\Delta}, \tau=0.2 ; \boldsymbol{O}, \tau=0.5 ; \boldsymbol{\square}, \tau=1$.

behaviour for all values of $\tau$. Table 2 gives these packing fractions for different $\tau$ and system sizes. If we assume naively that the dominant system size dependence of the percolation threshold goes as $1 / N$, we can obtain an estimate of the percolation threshold in an infinite system by simple extrapolation. As can be seen from figure 13 , such an extrapolation procedure is compatible with the available data, except perhaps for $\tau=0 \cdot 5$.

We can compare the Monte Carlo estimates for the percolation threshold with the theoretical predictions of [10]. In figure 14 the present estimates are compared with the 'Percus-Yevick' percolation line. Note that there are pronounced differences between the simulation results and the predictions of the analytical theory. This is hardly surprising: after all, the percolation transition of a system of hard spheres $(\tau \rightarrow \infty)$ will be at random close packing. In contrast, the Percus-Yevick-like approximation of [10] predicts percolation at the unphysical value $\phi=1$. And at low $\tau$ values the PY theory predicts percolation at $\phi=0.0$ for every value lower

Table 2. Percolation threshold as a function of the system size. $\tau$ denotes the 'temperature' and $N$ the number of particles of the systems. The last column gives the extrapolated values for $N \rightarrow \infty$.

\begin{tabular}{lcccc}
\hline & & $N$ & & \\
$\tau$ & 32 & 108 & 256 & Extr. \\
\hline 0.1 & 0.149 & $0 \cdot 141$ & 0.141 & 0.139 \\
$0 \cdot 15$ & 0.189 & 0.183 & 0.180 & 0.179 \\
0.2 & 0.223 & 0.217 & 0.217 & 0.216 \\
0.5 & 0.318 & 0.316 & 0.319 & 0.318 \\
1 & 0.403 & 0.393 & 0.394 & 0.391 \\
\hline
\end{tabular}


than $\tau=1 / 12$. It is therefore hardly surprising that the MC estimate for the percolation line is shifted with respect to the predictions of the analytical theory: at high $\tau$ to lower values of $\phi$, and at low $\tau$ to higher values of $\phi$.

For comparison, the results of Seaton and Glandt [13] are also shown in figure 14. As can be seen from this figure, there appears to be no systematic difference between our results and those of [13], even though they were obtained using different algorithms. Of couse, the present algorithm allows us to study percolation at much higher densities than is possible with the approach of Seaton and Glandt.

As a consequence, the MC percolation line appears to intersect the liquid-vapour coexistence curve. Within the two-phase region the percolation threshold is difficult to locate, due to a large scatter in the data points. The error bars in figure 14 indicate the minimum and maximum packing fractions, between which a transition from totally unpercolated to completely percolated takes place. Actually, what we are probing is not really a two-phase region, but rather a homogeneous metastable fluid which is stabilized by the large excess free energy needed to create a gas-liquid interface. It may seem that the study of such an artificial homogeneous system is only of academic interest. However, we shall argue below, that percolation in this metastable state has important consequences for the phase separation in real clustering colloids.

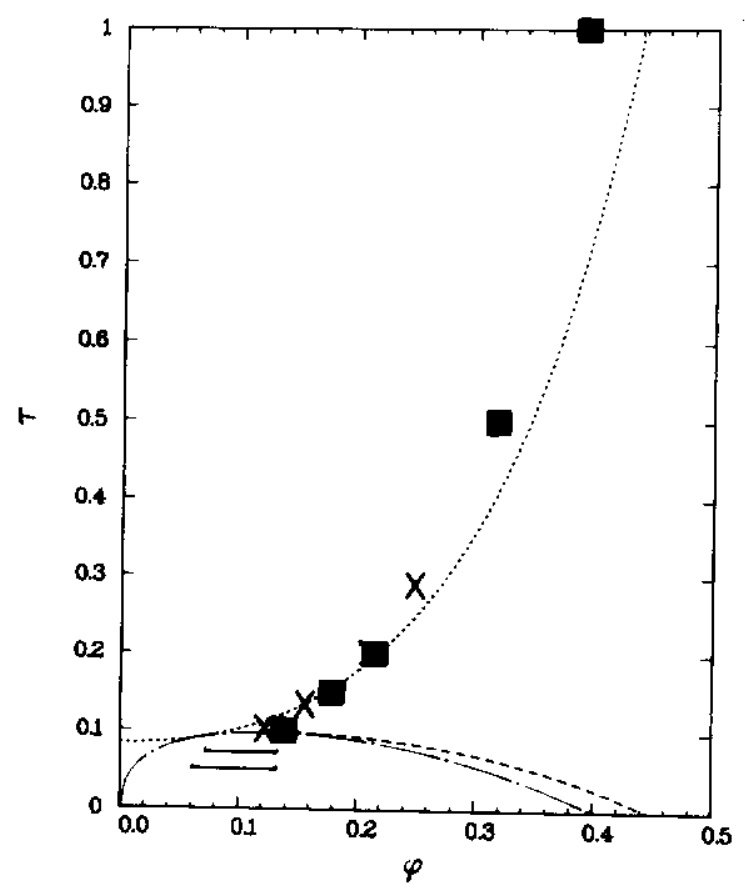

Figure 14. Percolation line and spinodal line. Horizontal axis: packing fraction; vertical axis: the 'temperature' $\tau$. The dotted line represents the PY prediction of the percolation line. Squares give the positions of the percolation thresholds, obtained in our work, after extrapolation to $1 / N \rightarrow 0$. Crosses represent data from $[12,13]$. The horizontal bars give the positions of percolation thresholds in the metastable gas phase. The dashed-dotted line borders the region, where the PY approximation has no real solutions, while the dashed line represents the spinodal line in the PY approximation. Note that for packing fractions smaller than the critical packing fraction, the spinodal line lies inside the region, where the PY approximation has no real solutions. 
In experiments on phase separation of dispersions of coated silica particles, which, to a first approximation may be considered an experimental realization of sticky spheres, Jansen et al. [21] observed that the two phase region appeared to have an anomalous shape. While the density of the dilute phase decreased upon lowering the temperature, as is to be expected, the density of the condensed phase also appeared to decrease. This is different from what is observed in simple onecomponent liquids, and is also not expected on basis of, for instance, the PercusYevick theory for sticky spheres. The present Monte Carlo simulations suggest that what was observed in the experiments of [21] was not the equilibrium liquid-vapour line, but rather the separation of the dispersion into a vapour phase and a metastable glassy phase. Such a glassy phase was often found in the Monte Carlo simulations. It is not expected to have the density of the rather tenuous, ramified system at the percolation threshold. Rather, we expect that the mechanical stability of such a glassy phase is determined by the 'rigidity percolation' threshold, which should be located at somewhat lower $\tau /$ higher $\phi$. Unfortunately, we were not able to extract more precise information about the location of the rigidity percolation line from our simulation data.

The authors like to thank Dr C. G. de Kruif and Dr J. G. H. Joosten for many fruitful discussions. This research was supported by the Netherlands Foundation for Chemical Research (SON) with financial aid from the Netherlands Organization for the Advancement of Pure Research (ZWO).

\section{Appendix}

\section{Monte Carlo algorithm for three-dimensional sticky spheres}

In this Appendix we describe the algorithm to change the number of bonds in three dimensions. We start by randomly choosing a particle. This particle is the centre of a sphere with radius $\Delta$, in which we randomly choose a point. This point is the centre of a second sphere, again with radius $\Delta$. In this second sphere the displacement will take place. The reason for this choice of the test-sphere is explained in $\S 3$.

To perform the actual displacements, the concept of the effective volume is essential. The effective volume in configuration-space of a particular type of bond is the volume in configuration-space, which covers all the realizations of that particular type of bond inside the test-sphere (or hypersphere in $N$ dimensions). A generalized expression for the effective volume of $b$ bonds of the test particle with other particles is

$$
V_{\text {eff }}(b)=\sum_{i}\left(\frac{\sigma}{12 \tau}\right)^{b} \int d q_{i}
$$

$d q_{i}$ represents all the degrees of freedom, remaining after making the bond(s). In equation (A 1) we have ignored all possible excluded volume effects. The summation is over all combinations of the test particle with the other particles which can realize that particular bond type.

In detail, the effective volume associated with zero bonds is just the volume of the test sphere. The effective volume of one bond is calculated as follows: consider one particular particle, with which the test-particle can form one bond. In principle it can be placed anywhere on the surface of the second particle, ignoring for the 
moment the boundaries of the test-sphere. So the effective volume for a single bond is found by integrating over the surface of a sphere

$$
V_{\mathrm{eff}}^{(1)}=\left(\frac{\sigma}{12 \tau}\right) \int_{0}^{\pi} \int_{0}^{2 \pi} \sin \theta d \theta d \phi=\frac{4 \pi \sigma^{3}}{12 \tau}
$$

Here we used the potential from $\S 2$.

Alternatively, bonds can be formed between the test particle and two other particles simultaneously. A necessary condition is that the distance between these two particles is less than twice the diameter of a particle. In that case the testparticle can be placed on a circle formed by all the points which lay on a distance $\sigma$ of both particles. The effective volume, associated with this double-bond is

$$
V_{\mathrm{eff}}^{(2)}=\left(\frac{\sigma}{12 \tau}\right)^{2} \int_{0}^{2 \pi} r d \phi,
$$

with $r$ the radius of the circle, where once again we have ignored the finite size of the test-sphere.

For the fourth possibility, the triple bond-type, the effective volume is given by

$$
V_{\text {eff }}^{(3)}=\left(\frac{\sigma}{12 \tau}\right)^{3} \frac{1}{\left|\mathbf{e}_{1} \cdot\left(\mathbf{e}_{2} \times \mathbf{e}_{3}\right)\right|}
$$

$\mathbf{e}_{1}, \mathbf{e}_{2}$ and $\mathbf{e}_{3}$ are the unit vectors, along the directions of the vectors joining the centre of the test particle to the centres of the other three particles, that are involved in the bond. Note that the direct making (and breaking) of triple bonds in nontetrahedral configurations is possible in the present algorithm, but not in the one employed by Seaton and Glandt [18]. Although this difference should influence the relative speed of the two algorithms, it does not affect the final results.

In equations (A 2)-(A 4) we have ignored the boundaries of the test-sphere. In the actual situation we calculate that part of the effective volume that is situated inside the test-sphere. Such calculations are relatively easy if trial moves are attempted in a spherical rather than a cubic volume.

In our Monte Carlo simulation we attempted to move particles successively. However, if a chosen particle was bound to four or more other particles, this particle was not moved, because of the demand of microscopic reversibility. After choosing a particle, we first made a list of all the particles, with which the test-particle could form a single bond within the test-sphere. From this list we select all pairs, with which the test-particle could form two bonds and finally we selected from the list of pairs those triplets, which could form a triple bond with the test-particle.

For each bond or set of bonds we calculated the associated effective volume, denoted by $V_{\text {eff }}^{(i)}$. Then we selected a particular configuration with a probability $P(i)$ given by

$$
P(i)=\frac{V_{\text {efftot }}^{(i)}}{\sum_{i=0}^{3} V_{\text {effot }}^{(i)}}
$$

In the second part of the Monte Carlo step, we attempted to move the test-particle to a random position in the selected subspace, i.e. a random displacement inside the test-sphere in the case of zero bonds, a random position at the 
surface of the second particle in the case of one bond, a random position on the circle in the case of a two bond and a random choic: of the two positions in the case of a three bond, of course with the restriction that these positions had to be inside the test-sphere.

After choosing a position, we tested for hard-core overlap and reject the trial move, if such an overlap was detected.

\section{References}

[1] BARKer, J. A., and Henderson, D., 1976, Rev. mod. Phys., 48, 587.

[2] Alder, B. J., and Wainwright, T. E., 1957, J. chem. Phys., 27, 1208.

[3] Ramakrishnan, T. V., and YussoufF, M., 1977, Solid St. Commun., 21, 389; 1979, Phys. Rev. B, 19, 2775. For a recent review, see: HAYMET, A. D. J., and OxTOBY, D. W., 1986, J. chem. Phys., 84, 1769.

[4] BaXTeR, R. J., 1968, J. chem Phys., 49, 2770.

[5] Watts, R. O., Henderson, D., and BaXter, R. J., 1971, Adv. chem. Phys., 21, 421.

[6] Barboy, B., 1974, J. chem. Phys., 61, 3194.

[7] Barboy, B., 1975, Chem. Phys., 11, 357.

[8] Smithline, S., and Haymet, A. D., 1985, J. chem. Phys., 83, 4103.

[9] Chan, D. Y. C., Pailthorpe, B. A., McGaskill, J. S., Mrtchell, P. J., and Ninham, B. W., 1979, J. colloid. interf. Sci., 72, 27.

[10] Chiew, Y. C., and Glandt, E. D., 1983, J. Phys. A, 16, 2599.

[11] Seaton, N. A., and GlandT, E. D., 1986, J. chem. Phys., 84, 4595.

[12] Seaton, N. A., and Glandt, E. D., 1987, PhysicoChem. Hydrodyn., 9, 369.

[13] Seaton, N. A., and GlandT, E. D., 1987, J. chem. Phys., 86, 4668.

[14] Post, A. J., and GlandT, E. D., 1986, J. chem. Phys., 84, 4585.

[15] Rotenberg, A., 1965, J. chem. Phys., 43, 1198.

[16] Hansen, J. P., and MCDonald, I. R., Theory of Simple Liquids, first edition (Academic Press), p. 119.

[17] Cummings, P. T., Perram, J. W., and Smith, E. R., 1976, Molec. Phys., 31, 535.

[18] Seaton, N. A., and Glandt, E. D., 1987, J. chem. Phys., 87, 1785.

[19] Cerjan, C., and Bagchi, B., 1985, Phys. Rev. A, 31, 1647.

[20] Coniglio, A., Angelis, U. De, Forlani, A., and Lauro, G., 1977, J. Phys. A, 10, 219.

[21] JANSEN, J. W., KRUIF, C. G. DE, and VRY, A., 1986, J. Coll. Interf. Sci., 114, 471; 1986, Ibid., 114, 481; 1986, Ibid., 114, 492; 1986, Ibid., 114, 501. 